The use of score tests for inference on variance components

Peer-reviewed author version

VERBEKE, Geert \& MOLENBERGHS, Geert (2003) The use of score tests for inference on variance components. In: Biometrics, 59(2). p. 254-262.

DOI: $10.1111 / 1541-0420.00032$

Handle: http://hdl.handle.net/1942/415 


\title{
The Use of Score Tests for Inference on Variance Components
}

\author{
Geert Verbeke* Geert Molenberghs $^{\dagger}$
}

\begin{abstract}
Whenever inference for variance components is required, the choice between onesided and two-sided tests is crucial. This choice is usually driven by whether or not negative variance components are permitted. For two-sided tests, classical inferential procedures can be followed, based on likelihood ratios, score statistics, or Wald statistics. For one-sided tests, however, one-sided test statistics need to be developed, and their null distribution derived. While this has received considerable attention in the context of the likelihood ratio test, there appears to be much confusion about the related problem for the score test. The aim of this paper is to illustrate that classical (two-sided) score test statistics, frequently advocated in practice, cannot be used in this context, but that well-chosen one-sided counterparts could be used instead. The relation with likelihood ratio tests will be established, and all results are illustrated in an analysis of continuous longitudinal data using linear mixed models.
\end{abstract}

Keywords: Boundary condition; Likelihood ratio test; Linear mixed model; Onesided test; Score test; Variance component.

\section{Introduction}

In a variety of applied statistical problems, there is a need for inference on variance components. This includes a variety of applied fields, for example, random-effects ANOVA models (Nelder 1954), linear mixed models (Verbeke and Molenberghs 2000), generalized linear and non-linear (mixed) models (Jacqmin-Gadda and Commenges 1995), overdispersion (Cox 1983, Smith and Heitjan 1993, Hines 1997, Lu 1997), clustering (Britton 1997) and homogeneity in stratified analyses (Liang 1987).

\footnotetext{
*Biostatistical Centre, Catholic University of Leuven, U.Z. St.-Rafaël, Kapucijnenvoer 35, B-3000 Leuven, Belgium

${ }^{\dagger}$ Biostatistics, Center for Statistics, Limburgs Universitair Centrum, tUL, Universitaire Campus, B-3590 Diepenbeek, Belgium
} 
To fix ideas, we will focus on the setting of a relatively simple linear mixed model, the so-called random-intercepts model:

$$
Y_{i j}=\boldsymbol{x}_{i j}^{\prime} \boldsymbol{\beta}+b_{i}+\varepsilon_{i j},
$$

where $Y_{i j}$ is the response for member $j=1, \ldots, n_{i}$ of cluster $i=1, \ldots, N, \boldsymbol{x}_{i j}$ is a vector of known covariate values, $\boldsymbol{\beta}$ is a vector of unknown regression coefficients, $b_{i} \sim N\left(0, \tau^{2}\right)$ is a cluster-specific random effect, assumed to be independently distributed from the residual error components $\varepsilon_{i j} \sim N\left(0, \sigma^{2}\right)$. Classical inferential procedures are based on the likelihood of the marginal model, obtained by integrating (1) over the random effects. Grouping the $Y_{i j}$ into a vector $\boldsymbol{Y}_{i}$ and assembling the rows $\boldsymbol{x}_{i j}^{\prime}$ into a matrix $X_{i}$, this marginal distribution is

$$
\boldsymbol{Y}_{i} \sim N\left(X_{i} \boldsymbol{\beta}, \tau^{2} J_{n_{i}}+\sigma^{2} I_{n_{i}}\right),
$$

in which $I_{n_{i}}$ denotes the identity matrix of dimension $n_{i}$, and where $J_{n_{i}}$ equals the $n_{i} \times n_{i}$ matrix containing only ones.

Regarding the variance component $\tau^{2}$ in the above model, one can take two views. In the first view, where the focus is entirely on the resulting marginal model (2), negative values for $\tau^{2}$ are perfectly acceptable (Nelder 1954, Verbeke and Molenberghs 2000, Sec. 5.6.2), since this merely corresponds to the occurrence of negative within-cluster correlation $\rho=\tau^{2} /\left(\tau^{2}+\sigma^{2}\right)$. This might occur, for example, in a context of competition such as when littermates compete for the same food resources. In such a case, the only requirement is that $\tau^{2}+\sigma^{2}>0$, for $V_{i}=\tau^{2} J_{n_{i}}+\sigma^{2} I_{n_{i}}$ to be a positive definite, marginal covariance matrix. Further discussions on negative variance components can be found in Thompson (1962) and Searle, Casella and McCulloch (1992). In the second view, when the link between the marginal model (2) and its generating hierarchical model (1) is preserved, thereby including the concept of random effects $b_{i}$ and perhaps even requiring inference for them, it is imperative to restrict $\tau^{2}$ to nonnegative values.

The first situation, which we will term the unconstrained case, is standard regarding inference 
for the variance component $\tau^{2}$. In the second situation (the constrained case), however, one typically needs one-sided tests of the null-hypothesis

$$
H_{0}: \tau^{2}=0 \quad \text { versus } \quad H_{A 1}: \tau^{2}>0
$$

As the null-hypothesis is now on the boundary of the parameter space, classical inference no longer holds, appropriate tailored test statistics need to be developed, and the corresponding (asymptotic) null distributions derived.

While this has received considerable attention in the case of the likelihood ratio test (Self and Liang 1987, Stram and Lee 1994, 1995), there is still much confusion about the related problem for the score test. For some uses of a score test in boundary situations, see Liang (1987), Lin (1997), Gray (1995), Jacqmin-Gadda and Commenges (1995), Dean and Lawless (1989), Dean (1992), Dean, Ugarte and Militino (2001), Gueorguieva (2001), Militino, Ugarte and Dean (2001), Smith and Heitjan (1993). Some authors implicitly take the unconstrained, two-sided view, with a few happy exceptions who explicitly adopt such a view (Paul and Islam 1995). Jacqmin-Gadda and Commenges (1995), Lin (1997), le Cessie and van Houwelingen (1995), and Dean, Ugarte, and Militino (2001) do not explicitly specify the alternative model, thereby implicitly assuming two-sided alternatives, while clearly being in a one-sided setting. We hope to illustrate that, when required by the scientific problem, a fully one-sided approach is both feasible and more appropriate. Silvapulle and Silvapulle (1995) have shown how a one-sided score test can be defined, both in the scalar as well as in the vector parameter case. Important related work is given in Hall and Præstgaard (2001). While these authors also focus on the restricted score tests in the context of mixed models, there are three important differences with our take on the problem. First, Hall and Præstgaard (2001) explicitly advocate the use of restricted score tests, thereby improving upon earlier work (Lin 1997) in terms of efficiency. We point out that the choice between a constrained/unconstrained setting should be tightly linked to a constrained/unconstrained alternative space. Second, since our score test statistics follow from the work of Silvapulle and Silvapulle (1995), their analytic forms are 
slightly different from those of Hall and Præstgaard (2001). Indeed, based on the results of Silvapulle and Silvapulle (1995), who showed that the asymptotic equivalence of the likelihood ratio and score tests holds, also in the constrained case, the null distribution of the one-sided score tests will be derived. Finally, we put a lot of emphasis on the extension of the wellknown asymptotic equivalence of the likelihood ratio and score tests to the constrained case, as follows from Silvapulle and Silvapulle (1995). We will argue that based on this equivalence, the researcher has full choice between both testing procedures, and moreover, opting for a constrained likelihood ratio tests has many computational advantages in practice. Emphasis will be on intuitive explanation of the theoretical results, rather than on mathematical details.

In Section 2, we continue with our initial model (1), and we will show how one-sided likelihood ratio and score tests can be constructed, and the corresponding asymptotic null distribution will be derived heuristically. Afterwards, in Sections 3 and 4, more general results will be discussed for the likelihood ratio test and for the score test, respectively. Note that our aim is not to argue for or against score tests, but rather show how to properly use one-sided score tests for variance components. Computational issues are discussed in Section 5. In Section 6, the results will be illustrated in an analysis of continuous longitudinal measurements, using linear mixed models, where the need for random effects is to be tested. Finally, Section 7 summarizes the main results.

\section{The Random-intercepts Model}

To introduce our ideas in a simple but generic setting, we continue the discussion of the random-intercepts model (1). Under the unconstrained parameterization, i.e., the model under which negative values for $\tau^{2}$ are allowed, classical inferential tools are available for testing the general two-sided hypothesis

$$
H_{0}: \tau^{2}=0 \quad \text { versus } \quad H_{A 2}: \tau^{2} \neq 0
$$


Wald, likelihood ratio, and score tests are then asymptotically equivalent, and the asymptotic null distribution is well known to be $\chi_{1}^{2}$ (Cox and Hinkley 1990). Under the constrained model, i.e., the model where $\tau^{2}$ is restricted to the non-negative real numbers, the one-sided hypothesis (3) is the only meaningful one.

Appropriate test statistics can now be obtained as follows. Suppressing dependence on the other parameters, let $\ell\left(\tau^{2}\right)$ denote the log-likelihood, as a function of the random-intercepts variance $\tau^{2}$. Further, let $\widehat{\tau}^{2}$ denote the maximum likelihood estimate of $\tau^{2}$ under the unconstrained parameterization. We first consider the likelihood ratio test, with statistic:

$$
T_{L R}=2 \ln \left[\frac{\max _{H_{1 A}} \ell\left(\tau^{2}\right)}{\max _{H_{0}} \ell\left(\tau^{2}\right)}\right] .
$$

Two cases, graphically represented in Figure 1, can now be distinguished. Under Case A, $\widehat{\tau}^{2}$ is positive, and the likelihood ratio test statistic is identical to the one that would be obtained under the unconstrained parameter space for $\tau^{2}$. Hence, conditionally on $\widehat{\tau}^{2} \geq 0, T_{L R}$ has asymptotic null distribution equal to the classical $\chi_{1}^{2}$. Under Case B however, we have that, under $H_{1 A}$ as well as under $H_{0}, \ell\left(\tau^{2}\right)$ is maximized at $\tau^{2}=0$ yielding $T_{L R}=0$. Further note that, under $H_{0}$, both cases occur with $50 \%$ probability. Hence, the asymptotic null distribution of $T_{L R}$ is obtained from

$$
\begin{aligned}
P & \left(T_{L R}>c \mid H_{0}\right) \\
& =P\left(T_{L R}>c \mid H_{0}, \widehat{\tau}^{2} \geq 0\right) P\left(\widehat{\tau}^{2} \geq 0 \mid H_{0}\right)+P\left(T_{L_{R}}>c \mid H_{0}, \widehat{\tau}^{2}<0\right) P\left(\widehat{\tau}^{2}<0 \mid H_{0}\right) \\
& =\frac{1}{2} P\left(\chi_{1}^{2}>c\right)+\frac{1}{2} P\left(\chi_{0}^{2}>c\right),
\end{aligned}
$$

where $\chi_{0}^{2}$ denotes the distribution with all probability mass at 0 . Hence, the asymptotic null distribution of the one-sided likelihood ratio test statistic is a mixture of two chi-squared distributions, with degrees of freedom 0 and 1 , and with equal mixing proportions $1 / 2$. This was one of Stram and Lee's $(1994,1995)$ special cases. Note that, whenever $\widehat{\tau}^{2} \geq 0$, the observed likelihood ratio test statistic is equal to the one under the unconstrained model, but the $p$-value is half the size of the one obtained from the classical $\chi_{1}^{2}$ approximation to the null distribution. 
We now consider the score test. The usual form of the test statistic is given by

$$
T_{S}=\left[\left.\frac{\partial \ell\left(\tau^{2}\right)}{\partial \tau^{2}}\right|_{\tau^{2}=0}\right]^{2}\left[-\left.\frac{\partial^{2} \ell\left(\tau^{2}\right)}{\partial \tau^{2} \partial \tau^{2}}\right|_{\tau^{2}=0}\right]^{-1} .
$$

Nuisance parameters are suppressed from notation, and replaced by their MLE's. In the special case of (2) with $n_{i} \equiv n$, straightforward algebra produces:

$$
T_{S}=\frac{N n}{2} \frac{(C-1)^{2}}{2 n C-1}
$$

with

$$
C=\frac{1}{\sigma^{2}} \frac{1}{N n} \sum_{i=1}^{N}\left(\sum_{j=1}^{n} y_{i j}\right)^{2},
$$

in which $\sigma^{2}$ is replaced by its maximum likelihood estimate under the null hypothesis, $\widehat{\sigma}^{2}$ say. Without loss of generality, it is assumed that the fixed-effects parameters are zero. In the reverse case, $y_{i j}$ needs to be replaced by appropriate residuals.

Now, score test (4) implicitly assumes a two-sided alternative. Hence, the test statistic itself need to be redefined appropriately in order to be able to discriminate between positive and negative alternative values for $\tau^{2}$. The same two cases as for the likelihood ratio test can be considered (see Figure 1). Under Case A, $\widehat{\tau}^{2}$ is positive, and the positive score $\partial \ell\left(\tau^{2}\right) / \partial \tau^{2}$ at zero is evidence against $H_{0}$ in favor of the one-sided alternative $H_{A 1}$. Hence, (4) can be used as test statistic, provided that $\widehat{\tau}^{2} \geq 0$. This implies that, conditionally on $\widehat{\tau}^{2} \geq 0$ and under $H_{0}$, our test statistic asymptotically follows the classical $\chi_{1}^{2}$ distribution. Under Case $\mathrm{B}$, however, the score at $\tau^{2}=0$ is negative, and can therefore clearly not be used as evidence against $H_{0}$ in favor of $H_{A 1}$. Hence, whenever $\widehat{\tau}^{2}$ is negative, (4) is no longer meaningful as test statistic. Considering that a negative score at zero supports the null hypothesis, a meaningful test statistic is obtained from replacing (4) by

$$
T_{S}= \begin{cases}{\left[\left.\frac{\partial \ell\left(\tau^{2}\right)}{\partial \tau^{2}}\right|_{\tau^{2}=0}\right]^{2}\left[-\left.\frac{\partial^{2} \ell\left(\tau^{2}\right)}{\partial \tau^{2} \partial \tau^{2}}\right|_{\tau^{2}=0}\right]^{-1}} & \text { if } \widehat{\tau}^{2} \geq 0 \\ 0 & \text { if } \widehat{\tau}^{2}<0 .\end{cases}
$$

The corresponding asymptotic null distribution is now obtained from

$$
P\left(T_{S}>c \mid H_{0}\right)
$$




$$
\begin{aligned}
& =P\left(T_{S}>c \mid H_{0}, \widehat{\tau}^{2} \geq 0\right) P\left(\widehat{\tau}^{2} \geq 0 \mid H_{0}\right)+P\left(T_{S}>c \mid H_{0}, \widehat{\tau}^{2}<0\right) P\left(\widehat{\tau}^{2}<0 \mid H_{0}\right) \\
& =\frac{1}{2} P\left(\chi_{1}^{2}>c\right)+\frac{1}{2} P\left(\chi_{0}^{2}>c\right),
\end{aligned}
$$

which is identical to the null distribution derived earlier for the likelihood ratio test. This heuristic but insightful argument will be formalized and generalized in Section 4.

Note that in this scalar case, an equivalent test consists of appropriately standardizing the score function rather than embedding it in a quadratic form. The choice between cases $A$ and $\mathrm{B}$ then merely becomes a choice between classical two-sided versus one-sided $Z$-type test procedures.

\section{$3 \quad$ Likelihood Ratio Tests}

When the use of the likelihood-ratio test is envisaged, it is now well known that hypotheses such as (3) pose non-standard testing problems (Verbeke and Molenberghs 2000, pp. 64-73). Such problems have been known for a long time (Nelder 1954, Chernoff 1954). Using results of Self and Liang (1987) on nonstandard testing situations, Stram and Lee $(1994,1995)$ have been able to show that the asymptotic null distribution for the likelihood ratio test statistic for

testing hypotheses of the type (3) is often a mixture of chi-squared distributions rather than the classical single chi-squared distribution. For ANOVA models with independent random effects, this was already briefly discussed by Miller (1977). This complication cannot be relieved by considering alternative parameterizations for the variance components, contradicting the once popular but false belief that replacing covariance matrices by their Cholesky decomposition was able to turn the problem in a standard one. Indeed, under the null hypothesis, the Cholesky decomposition does not map 1 to 1 onto the original parameterization.

Stram and Lee $(1994,1995)$ discuss likelihood ratio tests for variance components in linear mixed models, which are generalizations of (1) to models with multiple, possibly correlated, 
random effects. These models typically appear in the analysis of continuous longitudinal data. Let $\boldsymbol{Y}_{\boldsymbol{i}}$ denote the $n_{i}$-dimensional vector of measurements available for subject $i, i=1, \ldots, N$. A general linear mixed model then assumes that $\boldsymbol{Y}_{\boldsymbol{i}}$ satisfies

$$
\boldsymbol{Y}_{\boldsymbol{i}}=X_{i} \boldsymbol{\beta}+Z_{i} \boldsymbol{b}_{\boldsymbol{i}}+\boldsymbol{\varepsilon}_{\boldsymbol{i}}
$$

in which $\boldsymbol{\beta}$ is a vector of population-average regression coefficients called fixed effects, and where $\boldsymbol{b}_{\boldsymbol{i}}$ is a vector of subject-specific regression coefficients. The $\boldsymbol{b}_{\boldsymbol{i}}$ describe how the evolution of the $i$ th subject deviates from the average evolution in the population. The matrices $X_{i}$ and $Z_{i}$ are $\left(n_{i} \times p\right)$ and $\left(n_{i} \times q\right)$ matrices of known covariates. The random effects $\boldsymbol{b}_{\boldsymbol{i}}$ and residual components $\varepsilon_{\boldsymbol{i}}$ are assumed to be independent with distributions $N(\mathbf{0}, D)$, and $N\left(\mathbf{0}, \sigma^{2} I_{n_{i}}\right)$, respectively. Inference for linear mixed models is based on maximum likelihood or restricted maximum likelihood estimation under the marginal model for $\boldsymbol{Y}_{\boldsymbol{i}}$, i.e., the multivariate normal model with mean $X_{i} \boldsymbol{\beta}$, and covariance $V_{i}=Z_{i} D Z_{i}^{\prime}+\sigma^{2} I_{n_{i}}$ (Laird and Ware 1982, Verbeke and Molenberghs 2000).

Similar to our simpler model (1), the marginal model does not require $D$ to be positive definite, while a random-effects interpretation of the model does, corresponding to the unconstrained and constrained parameterizations, respectively. As before, inference under the unconstrained model for the variance components in $D$ can be based on the classical chi-squared approximation to the null distribution for the likelihood ratio test statistic. Under the constrained model, Stram and Lee $(1994,1995)$ have shown that the asymptotic null distribution for the likelihood ratio test statistic for testing a null hypothesis which allows for $k$ correlated random effects versus an alternative of $k+1$ correlated random effects (with positive semi-definite covariance matrix $D_{k+1}$ ), is a mixture of a $\chi_{k}^{2}$ and a $\chi_{k+1}^{2}$, with equal probability $1 / 2$. For more general settings, e.g., comparing models with $k$ and $k+k^{\prime}\left(k^{\prime}>1\right)$ random effects, the null distribution is a mixture of $\chi^{2}$ random variables (Shapiro 1988, Raubertas, Lee, and Nordheim 1986), the weights of which can only be calculated analytically in a number of special cases. 


\section{Score Tests}

Our heuristic arguments in Section 2 have suggested that employment of score tests for testing variance components under the constrained parameterization requires replacing the classical score test statistic by an appropriate one-sided version. This is where the general theory of Silvapulle and Silvapulle (1995) on one-sided score tests proves very useful. They consider models parameterized through a vector $\boldsymbol{\theta}=\left(\boldsymbol{\lambda}^{\prime}, \boldsymbol{\psi}^{\prime}\right)^{\prime}$, where testing a general hypothesis of the form

$$
H_{0}: \boldsymbol{\psi}=\mathbf{0} \quad \text { versus } \quad H_{A}: \boldsymbol{\psi} \in \mathcal{C}
$$

is of interest. In our context, the alternative parameter space $\mathcal{C}$ equals the nonnegative real numbers (e.g., when testing (3), or the set of positive semi-definite covariance matrices $D$ (e.g., when testing for the need of variance components in linear mixed models, Section 3). In general, Silvapulle and Silvapulle (1995) allow $\mathcal{C}$ to be a closed and convex cone in Euclidean space, with vertex at the origin. The advantage of such a general definition is that one-sided, two-sided, and combinations of one-sided and two-sided hypotheses are included.

Silvapulle and Silvapulle (1995) consider a general class of score-type test statistics. Applying their theory to our situation yields the following results. Let the log-likelihood function be denoted by $\ell(\boldsymbol{\theta})$. The associated score function equals

$$
\boldsymbol{S}_{N}(\boldsymbol{\theta})=\frac{\partial \ell}{\partial \boldsymbol{\theta}}
$$

Assume the existence of a nonsingular matrix $H(\boldsymbol{\theta})$ such that, for $N \rightarrow \infty$,

$$
(A 1): N^{-1 / 2} \boldsymbol{S}_{N}(\boldsymbol{\theta}) \stackrel{d}{\rightarrow} N(\mathbf{0}, H(\boldsymbol{\theta}))
$$

and, for all $a \geq 0$,

$$
(A 2): \sup _{\|\boldsymbol{h}\| \leq a}\left[N^{-1 / 2}\left\{\boldsymbol{S}_{N}\left(\boldsymbol{\theta}+N^{-1 / 2} \boldsymbol{h}\right)-\boldsymbol{S}_{N}(\boldsymbol{\theta})\right\}+H(\boldsymbol{\theta}) \boldsymbol{h}\right]=o_{p}(1) .
$$


Further, decompose $\boldsymbol{S}_{N}$ as $\boldsymbol{S}_{N}=\left(\boldsymbol{S}_{N \lambda}^{\prime}, \boldsymbol{S}_{N \psi}^{\prime}\right)^{\prime}$, let $H_{\lambda \lambda}(\boldsymbol{\theta}), H_{\lambda \psi}(\boldsymbol{\theta})$ and $H_{\psi \psi}(\boldsymbol{\theta})$ be the corresponding blocks in $H(\boldsymbol{\theta})$, and define $\boldsymbol{\theta}_{H}=\left(\boldsymbol{\lambda}^{\prime}, \mathbf{0}^{\prime}\right)^{\prime}$. $\boldsymbol{\theta}_{H}$ can be estimated by $\widehat{\boldsymbol{\theta}}_{H}=$ $\left(\widehat{\boldsymbol{\lambda}}^{\prime}, \mathbf{0}^{\prime}\right)^{\prime}$, in which $\widehat{\boldsymbol{\lambda}}$ is the maximum likelihood estimate of $\boldsymbol{\lambda}$, under $H_{0}$. Finally, let $\boldsymbol{Z}_{N}$ be equal to $\boldsymbol{Z}_{N}=N^{-1 / 2} \boldsymbol{S}_{N \psi}\left(\widehat{\boldsymbol{\theta}}_{H}\right)$. A one-sided score statistic can now be defined as

$$
T_{S}:=\boldsymbol{Z}_{N}^{\prime} H_{\psi \psi}^{1}\left(\widehat{\boldsymbol{\theta}}_{H}\right) \boldsymbol{Z}_{N}-\inf \left\{\left(\boldsymbol{Z}_{N}-\boldsymbol{b}\right)^{\prime} H_{\psi \psi}^{1}\left(\widehat{\boldsymbol{\theta}}_{H}\right)\left(\boldsymbol{Z}_{N}-\boldsymbol{b}\right) \mid \boldsymbol{b} \in \mathcal{C}\right\} .
$$

Note that our score statistic (5) derived heuristically for the random-intercepts model is a special case of the general test statistic (7). Indeed, when $\widehat{\tau}^{2}$ is positive, the score at zero is positive, and therefore in $\mathcal{C}$, such that the infimum in (7) becomes zero. For $\widehat{\tau}^{2}$ negative, the score at zero is negative as well and the infimum in (7) is attained for $\boldsymbol{b}=\mathbf{0}$, resulting in $T_{S}=0$.

It follows from Silvapulle and Silvapulle (1995) that, provided regularity conditions (A1) and (A2) hold, for $N \rightarrow \infty$, the likelihood ratio test statistic $T_{L R}$ satisfies $T_{L R}=T_{S}+o_{p}(1)$. Further, if the observed $T_{s}$ equals $t_{s}$, then the large-sample $p$-value equals

$$
p=\xi\left\{t_{s}, H_{\psi \psi}\left(\boldsymbol{\theta}_{I I}\right), \mathcal{C}\right\},
$$

where

$$
\xi(t, B, \mathcal{C})=\mathrm{P}\left[\boldsymbol{Z}^{\prime} B^{-1} \boldsymbol{Z}-\inf \left\{(\boldsymbol{Z}-\boldsymbol{b})^{\prime} B^{-1}(\boldsymbol{Z}-\boldsymbol{b}) \mid \boldsymbol{b} \in \mathcal{C}\right\} \geq t\right]
$$

and $\boldsymbol{Z} \sim N(0, B)$. Shapiro (1988, Eqs. 3.1 and 3.2) has shown that $1-\xi(t, B, \mathcal{C})$ equals a weighted sum of chi-squared probabilities. The results obtained by Stram and Lee (1994, 1995) for the linear mixed model are included in Shapiro's results. There are a few additional results available. For example, if the null hypothesis allows for $k$ uncorrelated random effects (with a diagonal covariance matrix $D_{k}$ ) versus the alternative of $k+k^{\prime}$ uncorrelated random effects (with diagonal covariance matrix $D_{k+k^{\prime}}$ ), the null distribution is a mixture of the form

$$
\sum_{m=0}^{k^{\prime}} 2^{-k^{\prime}}\left(\begin{array}{c}
k^{\prime} \\
m
\end{array}\right) \chi_{m}^{2}
$$

Shapiro (1988) shows that, for a broad number of cases, determining the mixture's weights is a complex and perhaps numerical task. 
The above results show that the equivalence of the score and likelihood ratio tests not only holds in the two-sided but also in the one-sided cases. At the same time, an appropriate definition of the one-sided score statistic is produced, and its asymptotic null distribution has been derived. In some cases, the analytic null distribution is easily obtained through the results of Stram and Lee $(1994,1995)$, summarized in Section 3, and the equivalence between the likelihood ratio and score tests.

Finally, it should be emphasized that all above results are valid provided that the conditions $(A 1)$ and $(A 2)$ are satisfied. In particular, $(A 2)$ requires that the score $\boldsymbol{S}_{N}$ exists in a sufficiently small neighborhood around $H_{0}$. For example, in our random-intercepts example (Section 2), it is crucial to have valid models for sufficiently small but negative values of $\tau^{2}$, even in the constrained setting. As a counterexample, if we were to test $H_{0}: \sigma^{2}=0$ versus the one-sided alternative $H_{A}: \sigma^{2}>0$ for the variance $\sigma^{2}$ in a univariate normal $N\left(0, \sigma^{2}\right)$ sample of size $N$, the score equals

$$
\boldsymbol{S}_{N}\left(\sigma^{2}\right)=\frac{\partial \ell}{\partial \sigma^{2}}=-\frac{N}{2 \sigma^{2}}+\frac{1}{2 \sigma^{4}} \sum_{i=1}^{N} y_{i}^{2},
$$

which, evaluated at $\sigma^{2}=0$, yields $+\infty$. Then, the above theory does not apply here as no negative values for $\sigma^{2}$ can ever yield a valid statistical model for our sample. Hence, in this example, condition $(A 2)$ is no longer satisfied.

\section{Computational Issues}

In this section, computations for likelihood ratio and score test statistics will be discussed, for the classical unconstrained as well as the constrained cases. The relative complexity of all four cases will be addressed. Here, we focus on the general concepts, while a particular example using the SAS procedures MIXED and NLMIXED is deferred to the Appendix.

In principle, calculation of the unconstrained likelihood ratio test statistic does not pose any 
specific complications, provided both the null and alternative models can be fitted with standard software, and both log-likelihood values at maximum are returned, minus twice the difference of which is then referred to the appropriate chi-squared distribution, to yield the $p$-value.

Even in the unconstrained case, the score test calculations are more involved than their likelihood ratio counterparts, since the first and second order derivatives of the alternative loglikelihood function are required, evaluated under the null hypothesis. These cannot easily be obtained in many standard packages without additional programming. Once these derivatives have been obtained, they are the straightforward building blocks for the calculation of the test statistic, while the $p$-value is obtained as in the previous case.

The constrained likelihood ratio test statistic can be obtained in the same way as in the unconstrained case, provided the constraints are properly imposed onto the alternative model. In many practical situations, this comes down to maximizing the likelihood under a positive definiteness constraint on a covariance matrix $D$ as, for example, in the linear mixed model setting. Several routes can be followed. Replacing $D$ by its Cholesky decomposition $\left(D=L^{\prime} L\right)$ and maximizing over $L$ rather than $D$ turns the constrained optimization into an unconstrained one. This route has been proposed by Lindstrom and Bates (1988). Note that the constrained testing problem is not turned into an unconstrained one, because the Cholesky decomposition does not map one-to-one onto the original parameterization, thus maintaining the need for an appropriate testing theory as developed in this article and by Hall and Præstgaerd (2001). Alternatively, a so-called barrier type approach can be followed, for example, by adding a penalty $a \log \{\operatorname{det}(D)\}$ to the log-likelihood function, for some pre-specified constant $a$. While a careful consideration of the relative merits of these and other approaches is important and interesting in its own right, it is beyond the scope of this paper.

The constrained score test statistic (7) is composed of two parts. The first term is identical 
to the unconstrained counterpart, while the second term involves a constrained minimization of the quadratic form $\left(\boldsymbol{Z}_{N}-\boldsymbol{b}\right)^{\prime} H_{\psi \psi}^{-1}\left(\widehat{\boldsymbol{\theta}}_{H}\right)\left(\boldsymbol{Z}_{N}-\boldsymbol{b}\right)$ which cannot always be done analytically. In such cases, additional software code needs to be written, invoking numerical constrained optimization routines.

In both constrained cases, $p$-value computation is given by (8) which is a weighted sum of chisquared probabilities, the weights of which are known analytically in special (but important) cases only.

\section{Application: The Rat Data}

Using a simple case study and a selected set of nested models, we illustrate (1) likelihood ratio as well as score tests, (2) under both one-sided and two-sided alternatives, and (3) in cases where a boundary estimate does and does not occur.

The data considered to this end are from a randomized longitudinal experiment, previously described and analyzed by Verdonck et al. (1998), in which 50 male Wistar rats were randomized to either a control group or one of the two treatment groups where treatment consisted of a low or high dose of the drug Decapeptyl, which is an inhibitor for testosterone production in rats. The primary aim of the study was to investigate the effect of the inhibition of the production of testosterone in male Wistar rats on their craniofacial growth. The treatment started at the age of 45 days, and measurements were taken every 10 days, with the first observation taken at the age of 50 days. One of the responses of interest was the height of the skull, measured as the distance (in pixels) between two well-defined points on X-ray pictures of the skull, taken after the rat has been anesthetized. The individual profiles are shown in Figure 2. Although rats were scheduled to be followed up to the age of 110 days, some drop out prematurely because they do not survive anaesthesia. In fact, while 50 rats have been 
randomized at the start of the experiment, only 22 of them survived the first 6 measurements, so measurements on only 22 rats are available in the way anticipated at the design stage.

As before, let $Y_{i j}$ denote the response taken at time $t_{j}$, for rat $i$. Verbeke and Lesaffre (1999) and Verbeke and Molenberghs (2000) proposed to model the subject-specific profiles shown in Figure 2 as linear functions of $t=\ln (1+($ Age -45$) / 10)$. More specifically, their model is of the form

$$
Y_{i j}= \begin{cases}\beta_{0}+b_{1 i}+\left(\beta_{1}+b_{2 i}\right) t_{i j}+\varepsilon_{i j}, & \text { if low dose } \\ \beta_{0}+b_{1 i}+\left(\beta_{2}+b_{2 i}\right) t_{i j}+\varepsilon_{i j}, & \text { if high dose } \\ \beta_{0}+b_{1 i}+\left(\beta_{3}+b_{2 i}\right) t_{i j}+\varepsilon_{i j}, & \text { if control. }\end{cases}
$$

Here, $\beta_{0}$ is the average response at the time of randomization, while $\beta_{1}, \beta_{2}$ and $\beta_{3}$ are the average slopes in the three different treatment groups. Further, the $b_{1 i}$ and $b_{2 i}$ are rat-specific intercepts and slopes, representing the natural heterogeneity between rats with respect to baseline values and with respect to evolutions over time, respectively. The above model is an example of linear mixed model (6). As in our introductory example we have that, strictly speaking, the marginal model does not require $D$ to be positive definite, as long as the resulting marginal covariance $V_{i}$ is. Hence, when testing for elements in $D$, two-sided tests can be employed. However, they then no longer allow the hierarchical interpretation of the model, i.e., the interpretation in which the variability in the data is believed to be generated from an underlying random-effects model as in (9). If underlying random effects are believed to be latently present, one-sided tests are required.

Several models can now be fitted and compared with one another. Table 1 summarizes some of the results obtained from fitting and comparing a series of models to the rat data. Model 1 assume independent repeated measures and does not include any random effects; its only variance component is the common variance $\sigma^{2}$. Model 2 includes random intercepts only and is therefore an example of (1), assuming all measurements $Y_{i j}$ within subject $i$ exhibit equal correlation and common variance. Finally, Models 3 and 4 include random linear timeeffects $b_{2 i}$ as well, which may (Model 3) or may not (Model 4) be correlated with the random 
intercepts $b_{1 i}$.

Table 1 shows the results of one- as well as two-sided, likelihood ratio as well as score tests, for model comparisons $2-1,3-2$, and $4-2$. Comparison $2-1$ is standard in the sense that, since the unconstrained estimate of the random-intercepts variance $d_{11}$ under Model 2 is positive, the one- and two-sided test statistics are identical, and only the null distribution is different. For comparison 3-2, the unconstrained estimate for the random-slopes variance $d_{22}$ is negative, yielding different one- and two-sided test statistics. For the score test, for example, the infimum in (7) is attained for $\boldsymbol{b}=0$, yielding zero as observed value for the one-sided test statistic. For comparison 4-2, the infimum in (7) needs to be calculated numerically, and was found to be equal to 1.93 , which is attained for $\boldsymbol{b}=(-0.801,0.187)^{\prime}$.

In the likelihood ratio case, one might be tempted to combine a constrained calculation of the test statistic with reference to the classical $\chi^{2}$ null distribution. This, however, would lead to $p$-values that are too large. Therefore, the null hypothesis would be accepted too often, resulting in incorrectly simplifying the covariance structure of the model, which may seriously invalidate inferences, as shown by Altham (1984).

\section{Concluding Remarks}

Whenever inference for variance components is of interest, the choice between one-sided or two-sided tests is crucial, depending on whether negative variance components are deemed meaningful or not. For two-sided tests, classical inferential procedures can be followed, based on likelihood ratios, score statistics, or Wald statistics, which have the same asymptotic null distributions. For one-sided tests, however, one-sided test statistics need to be developed, and their null distribution derived. In contrast to the case of likelihood ratio tests, this has thus far not received much attention in the score-test case. Moreover, there seems to be a 
lot of confusion as to whether or not classical score tests are applicable in this setting. Using heuristic arguments in the context of a simple linear random-effects model, we have shown why those test statistics are not appropriate for testing one-sided hypotheses, and how one-sided versions can be obtained. Then, the general theory of Silvapulle and Silvapulle (1995) has been invoked to derive general one-sided score tests for variance components. Further, the well-known equivalence between two-sided score and likelihood ratio tests is shown to hold true for the one-sided counterparts as well.

In general, likelihood ratio tests as well as score tests are available for testing hypotheses about variance components, and both procedures are asymptotically equivalent, for one-sided as well as two-sided tests. The choice between one-sided and two-sided tests should be entirely driven by the scientific question, the data analyzed, the models fitted, and the interpretation of the parameters in those models. A frequently quoted justification for the use of score tests is that they do not require fitting the alternative model. However, currently available software easily allows pracitising statisticians to fit and compare a variety of models, containing many variance components. Moreover, whenever one-sided tests are of interest, the score test may require employing numerical optimization techniques for the calculation of the infimum in (7). Therefore, it cannot be our intention to advocate the broad use of score tests for the inference on variance components. Instead, the aim of this paper has been to enhance insight into the score test and to illustrate the use of score tests in this context.

We hope to have indicated that one either can take an unconstrained view and then no additional action is needed in case variance components are negative, or one takes a constrained view and then the inferential procedures should be such that proper constraints are imposed. In this sense, the statement made by Brown and Prescott $(1999$, p. 237): "The usual action when a negative variance component estimate is obtained for a random coefficient would be to refit the model with the random coefficient removed $(. .)$.$" , overlooks important issues and$ is therefore misleading. 


\section{Acknowledgments}

We gratefully acknowledge support from FWO-Vlaanderen Research Project "Sensitivity Analysis for Incomplete and Coarse Data" and Belgian IUAP/PAI network "Statistical Techniques and Modeling for Complex Substantive Questions with Complex Data". We are very grateful to David Cox for sharing his insights with us and for very helpful comments on an earlier version of this manuscript.

\section{Appendix}

Within the context of the case study, we will show how all four tests discussed in Section 5 can be implemented using the SAS procedures MIXED and NLMIXED (Version 8.2). In particular, we will focus on the comparison between Models 2 and 4.

Let us consider the likelihood ratio tests first. Fitting Model 4, with random intercepts and random slopes is typically done using the code (Verbeke and Molenberghs 2000, Ch. 8):

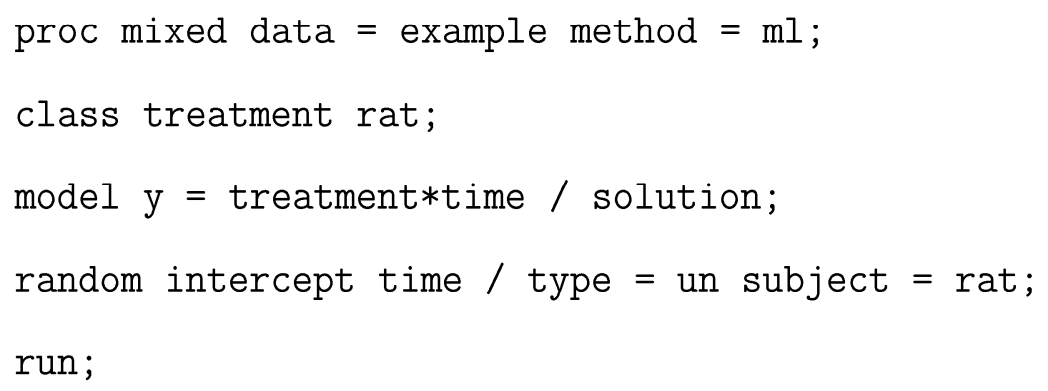

However, it should be noted the SAS procedure MIXED takes by default a constrained viewpoint which differs not only from the unconstrained situation, but is also different from the constrained formulation considered in this paper. Indeed, the only constraints imposed by SAS are for the variances to be nonnegative; the resulting covariance matrix can still fail 
to be positive semi-definite. A completely unconstrained model can be fitted by adding the 'nobound' option to the PROC MIXED statement. The likelihood ratio test statistic then follows from fitting Model 2 as well, which is obtained from deleting the variable time from the RANDOM statement.

Fitting both models under our constraints is done by replacing the 'type=un' option in the RANDOM statement of the initial program by 'type $=\mathrm{fa} 0(2)$ ' in Model 4 and 'type $=\mathrm{fa} 0(1)$ ' in Model 2.

Recall that, for the unconstrained score test, the first and second order derivates of the alternative log-likelihood need to be evaluated at the MLE's under the null model. We therefore first fit the null model using the SAS procedure MIXED, then store the parameter estimates in appropriately formatted SAS data sets, whereafter the derivatives under the alternative model are obtained using the NLMIXED procedure. The NLMIXED is particularly convenient since it easily produces the first and second order derivatives at the maximum likelihood estimates. To this end, the MLE's under the null are supplied to NLMIXED as starting values and the procedure is prevented from iterating. Practically, the results are obtained using the following code.

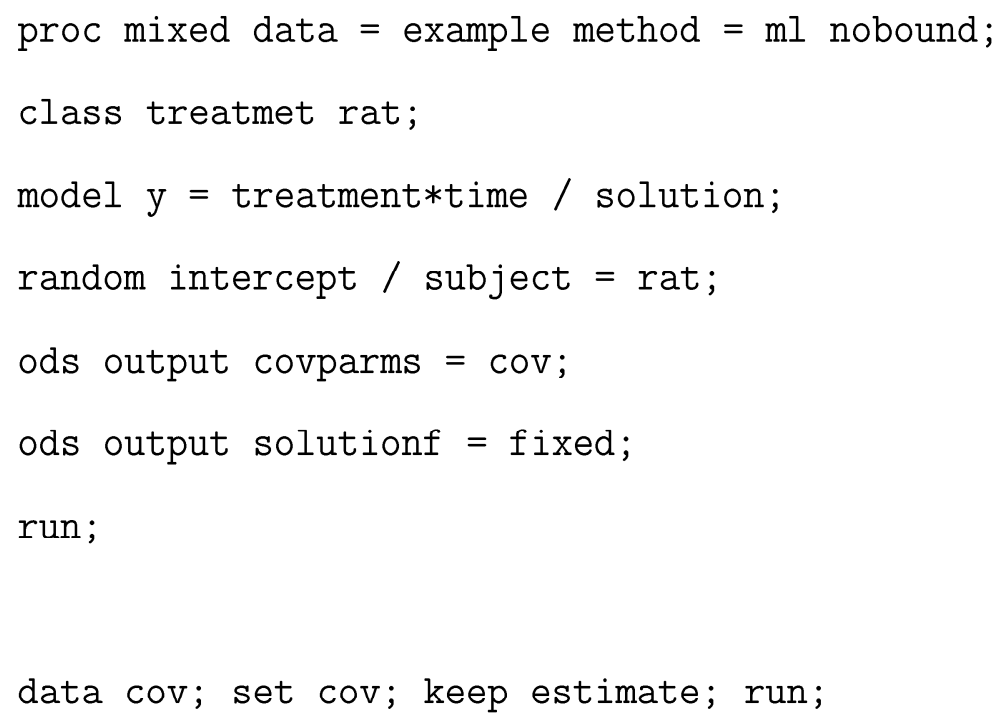




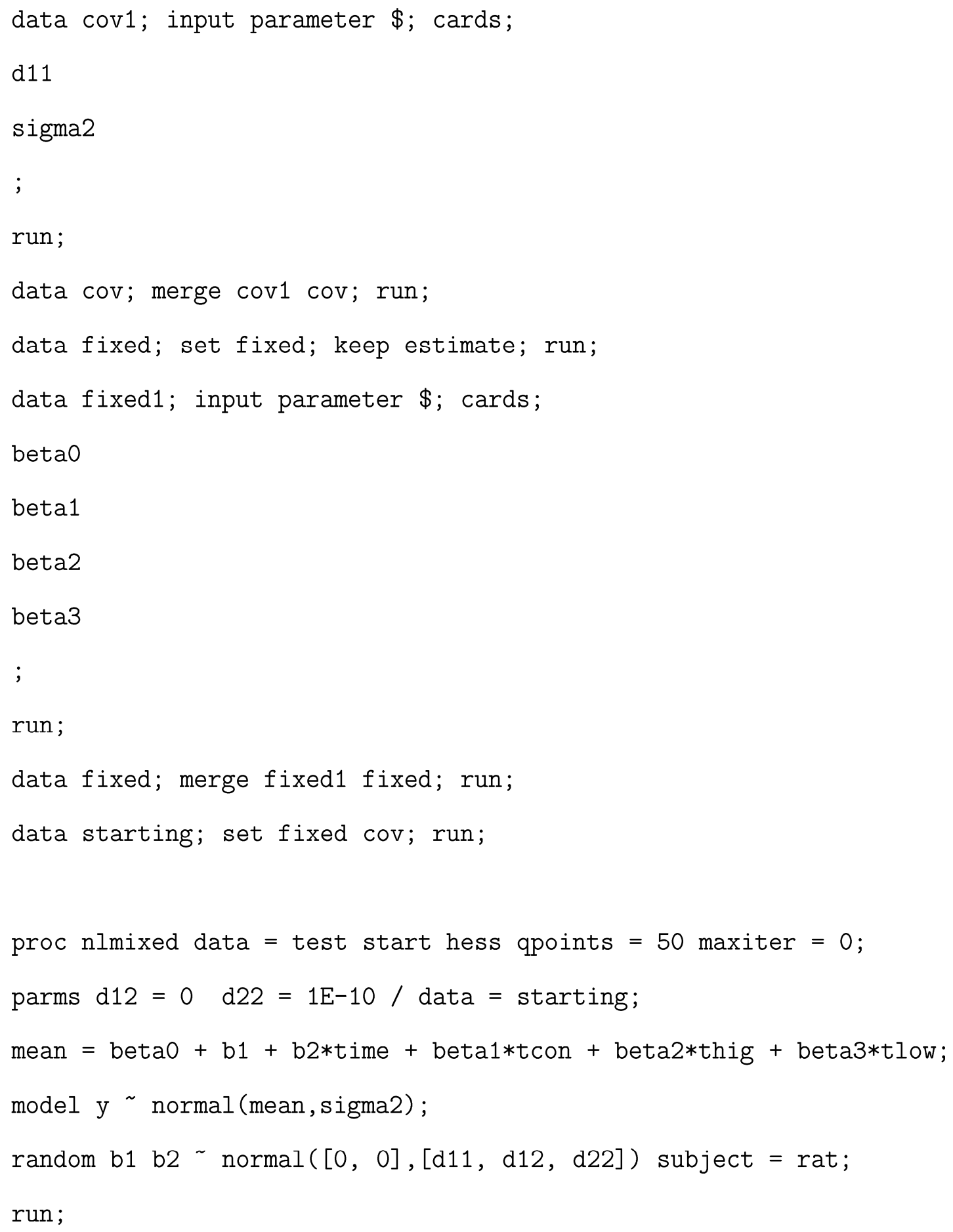

For the constrained case, the above program is maintained for the calculation of the first term in (7), except for the removal of the 'nobound' option from the PROC MIXED statement. To execute the constrained minimization of $\left(\boldsymbol{Z}_{N}-\boldsymbol{b}\right)^{\prime} H_{\psi \psi}^{-1}\left(\widehat{\boldsymbol{\theta}}_{H}\right)\left(\boldsymbol{Z}_{N}-\boldsymbol{b}\right)$, numerical optimization 
routines are often needed. While several routes could be followed, we have chosen to use the constrained optimization features of GAUSS. To this end, the constraints need to be written in the form of a procedure, with a second procedure containing the objective function. In our example, $\boldsymbol{Z}_{N}=(0.10219,-6.619967)^{\prime}$ and

$$
H_{\psi \psi}\left(\widehat{\boldsymbol{\theta}}_{H}\right)=\left(\begin{array}{cc}
16.5355 & -9.1388 \\
-9.1388 & 26.2642
\end{array}\right) .
$$

The code then becomes:

library co;

proc ineqp (b);

local d11, d12, d22, d;

$\mathrm{d} 11=3.4374 ;$

$\mathrm{d} 12=\mathrm{b}[1,$.$] ;$

$\mathrm{d} 22=\mathrm{b}[2,$.$] ;$

$d=(d 11 \sim d 12) \mid\left(d 12^{\sim} d 22\right)$;

$\operatorname{retp}(\operatorname{minc}(\operatorname{eigh}(d))-1 e-5)$;

endp;

_co_IneqProc=\&ineqp;

proc factor2(b);

local zn, hmat;

$\mathrm{zn}=\{0.10219,-6.619967\}$;

hmat $=\{16.5355-9.1388,-9.138826 .2642\}$;

$\operatorname{retp}((z n-b) ' * \operatorname{inv}($ hmat $) *(z n-b))$;

endp; 


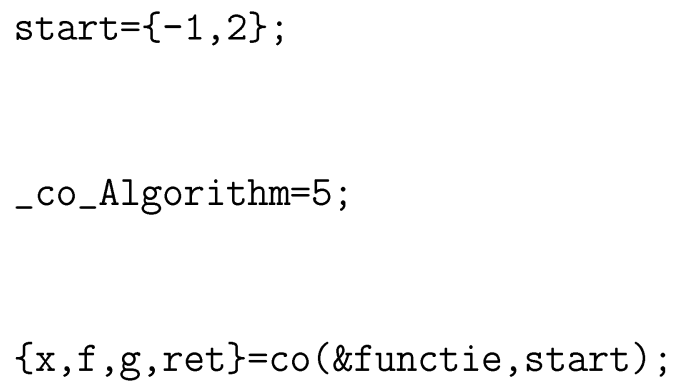

\section{References}

Altham, P.M.E. (1984). Improving the precision of estimation by fitting a model. Journal of the Royal Statistical Society, Series B 46, 118-119.

Britton, T. (1997). Tests to detect clustering of infected individuals within families. Biometrics 53, 98-109.

Brown, H. and Prescott, R. (1999). Applied Mixed Models in Medicine. Chichester: John Wiley.

Chernoff, H. (1954). On the distribution of the likelihood ratio. Annals of Matematical Statistics 25, 573-578.

Cox, D.R. (1983). Some remarks on overdispersion. Biometrika 70, 269-274.

Cox, D.R. and Hinkley, D.V. (1990). Theoretical Statistics. London: Chapman \& Hall.

Dean, C. (1992). Testing for overdispersion in Poisson and binomial regression models. Journal of the American Statistical Association 87, 451-457.

Dean, C. and Lawless, J.F. (1989). Tests for detecting overdispersion in Poisson regression models. Journal of the American Statistical Association 84, 467-472. 
Dean, C.B., Ugarte, M.D., and Militino, A.F. (2001). Detecting interaction between random region and fixed age effects in disease mapping. Biometrics 57, 192-202.

Gueorguieva, R. (2001). A multivariate generalized linear mixed model for joint modelling of clustered outcomes in the exponential family. Statistical Modelling 1, 177-193.

Gray, R.J. (1995). Tests for variation over groups in survival data. Journal of the American Statistical Association 90, 198-203.

Hall, D.B. and Præstgaard, J.T. (2001). Order-restricted score tests for homogeneity in generalised linear and nonlinear mixed models. Biometrika 88, 739-751.

Hines, R.J.O. (1997). A comparison of tests for overdispersion in generalized linear models. Journal of Statistical Computation and Simulation 58, 323-342.

Jacqmin-Gadda, H. and Commenges, D. (1995). Tests of homogeneity for generalised linear models. Journal of the American Statistical Association 90, 1237-1246.

Laird, N.M. and Ware, J.H. (1982). Random effects models for longitudinal data. Biometrics 38, 963-974.

le Cessie, S. and van Houwelingen, J.C. (1995). Testing the fit of a regression model via score tests in random effects models. Biometrics 51, 600-614.

Liang, K.-Y. (1987). A locally most powerful test for homogeneity with many strata. Biometrika 74, 259-264.

Lin, X. (1997). Variance component testing in generalised linear models with random effects. Biometrika 84, 309-326.

Lindstrom, M.J. and Bates, D.M. (1988). Newton-Raphson and EM algorithms for linear mixed-effects models for repeated-measures data. Journal of the American Statistical Association 83, 1014-1022. 
Lu, W.S. (1997). Score tests for overdispersion in Poisson regression models. Journal of Statistical Computation and Simulation 56, 213-228.

Militino, A.F., Ugarte, M.D., and Dean, C.B. (2001). The use of mixture models for identifying high risks in disease mapping. Statistics in Medicine 20, 2035-2049.

Miller, J.J. (1977). Asymptotic properties of maximum likelihood estimates in the mixed model of the analysis of variance. The Annals of Statistics 5, 746-762.

Nelder, J.A. (1954). The interpretation of negative components of variance. Biometrika 41, 544-548.

Paul, S.R. and Islam, A.S. (1995). Analysis of proportions in the presence of over- and under-dispersion. Biometrics 51, 1400-1410.

Raubertas, R.F., Lee, C.I.C., and Nordheim, E.V. (1986). Hypothesis tests for normal means constrained by linear inequalities. Communications in Statistics-Theory and Methods 15, 2809-2833.

Searle, S.R., Casella, G., and McCulloch, C.E. (1992). Variance Components. New York: John Wiley \& Sons.

Self, S.G. and Liang, K.Y. (1987). Asymptotic properties of maximum likelihood estimators and likelihood ratio tests under nonstandard conditions. Journal of the American Statistical Association 82, 605-610.

Shapiro, A. (1988). Towards a unified theory of inequality constrained testing in multivariate analysis. International Statistical Review 56, 49-62.

Silvapulle, M.J. and Silvapulle, P. (1995). A score test against one-sided alternatives. Journal of the American Statistical Association 90, 342-349.

Smith, P.J. and Heitjan, D.F. (1993). Testing and adjusting for departures from nominal disperson in generalized linear models. Applied Statistics 41, 31-41. 
Stram, D.O. and Lee, J.W. (1994). Variance components testing in the longitudinal mixed effects model. Biometrics 50, 1171-1177.

Stram, D.A. and Lee, J.W. (1995). Correction to: Variance components testing in the longitudinal mixed effects model. Biometrics 51, 1196.

Thompson, W.A., Jr. (1962). The problem of negative estimates of variance components. Annals of Mathematical Statistics 33, 273-289.

Verbeke, G. and Lesaffre, E. (1999). The effect of drop-out on the efficiency of longitudinal experiments. Applied Statistics 48, 363-375.

Verbeke, G. and Molenberghs, G. (2000). Linear mixed models for longitudinal data, Springer Series in Statistics, Springer-Verlag, New-York.

Verdonck, A., De Ridder, L., Verbeke, G., Bourguignon, J.P., Carels, C., Kuhn, E.R., Darras, V., and de Zegher, F. (1998). Comparative effects of neonatal and prepubertal castration on craniofacial growth in rats. Archives of Oral Biology 43, 861-871. 


\section{Captions}

Table 1. Rat data. Summary of the results of one- as well as two-sided, likelihood ratio as well as score tests for the comparison of a series of linear mixed models fitted to the rat data.

The estimate $\widehat{D}$ denotes the unconstrained maximum likelihood estimate for the matrix $D$ in the linear mixed model.

Figure 1. Graphical representation of two different situations, when developing one-sided tests for the variance $\tau^{2}$ of the random intercepts $b_{i}$ in model $(1)$.

Figure 2. Rat Data. Individual profiles for each of the treatment groups in the rat experiment separately. 


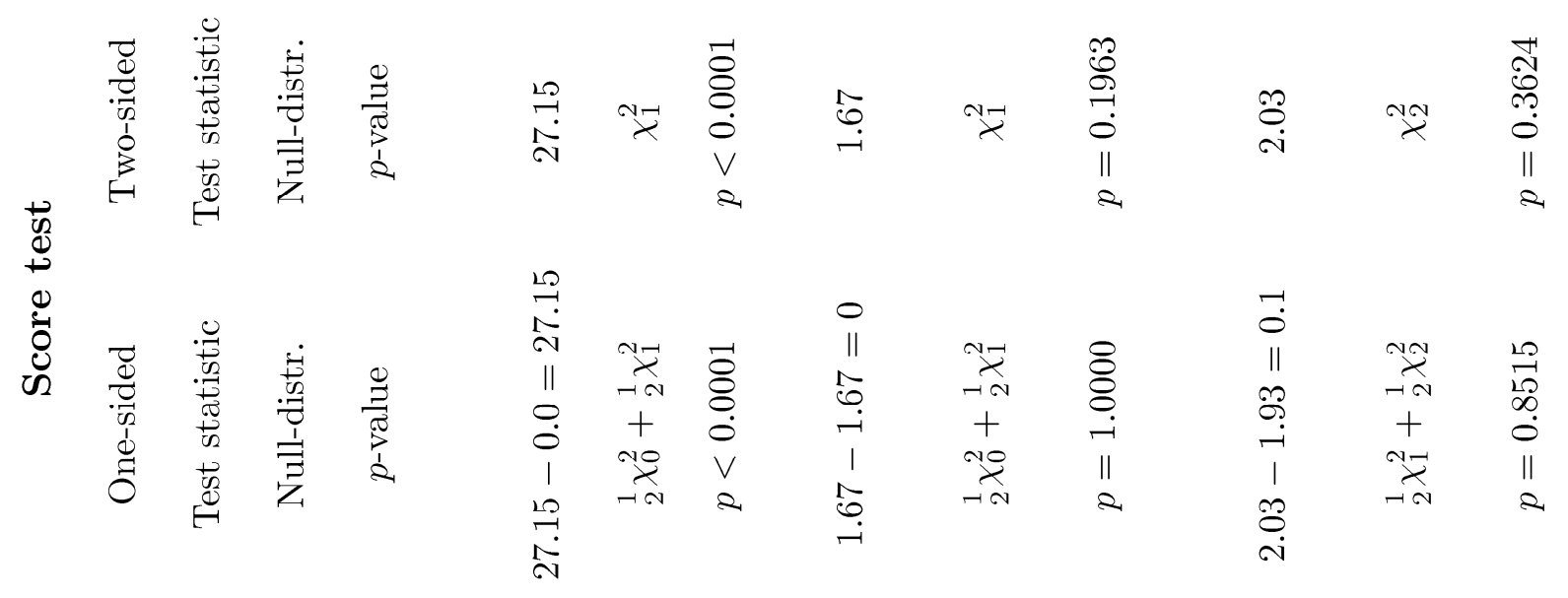
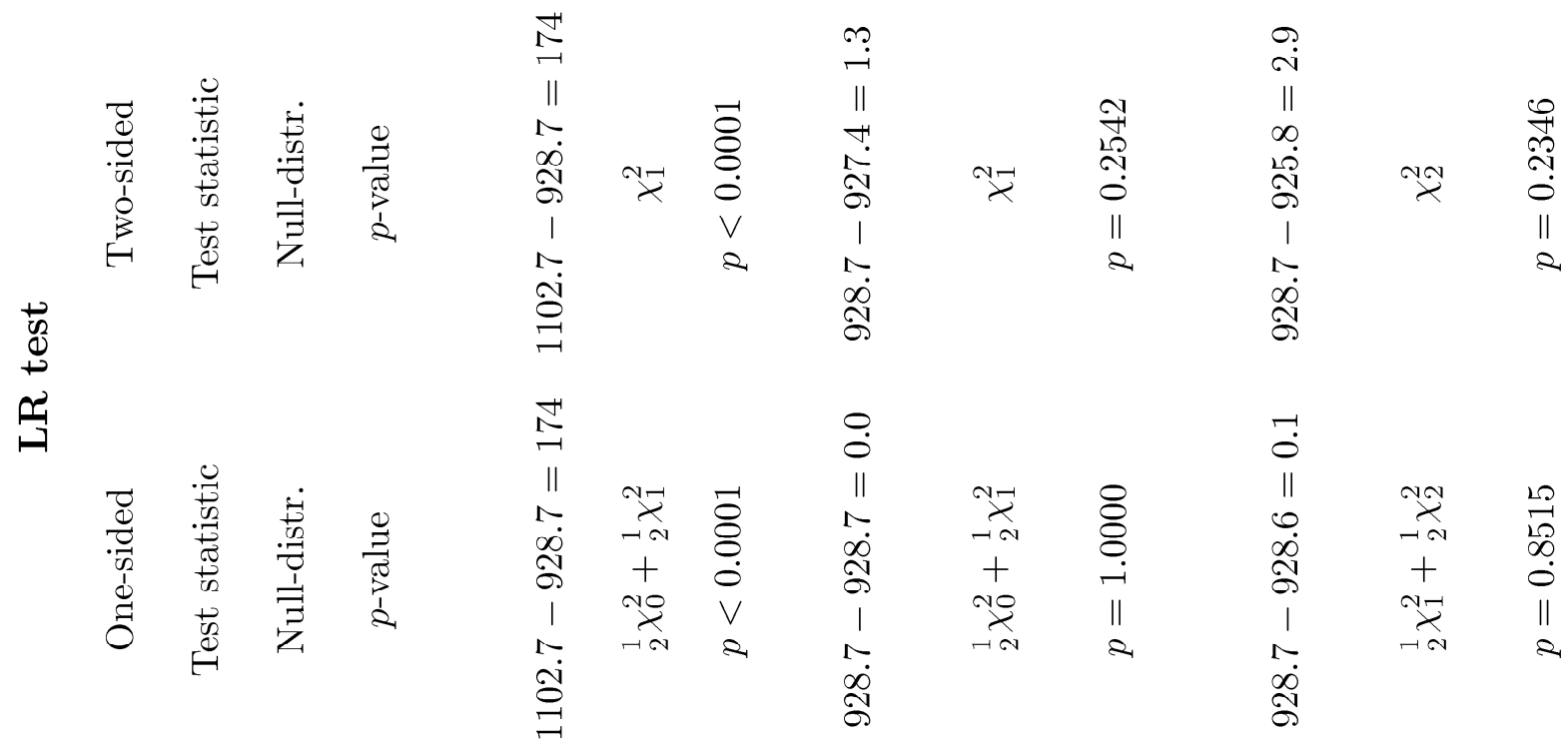

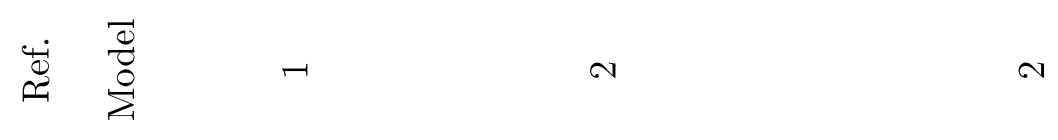

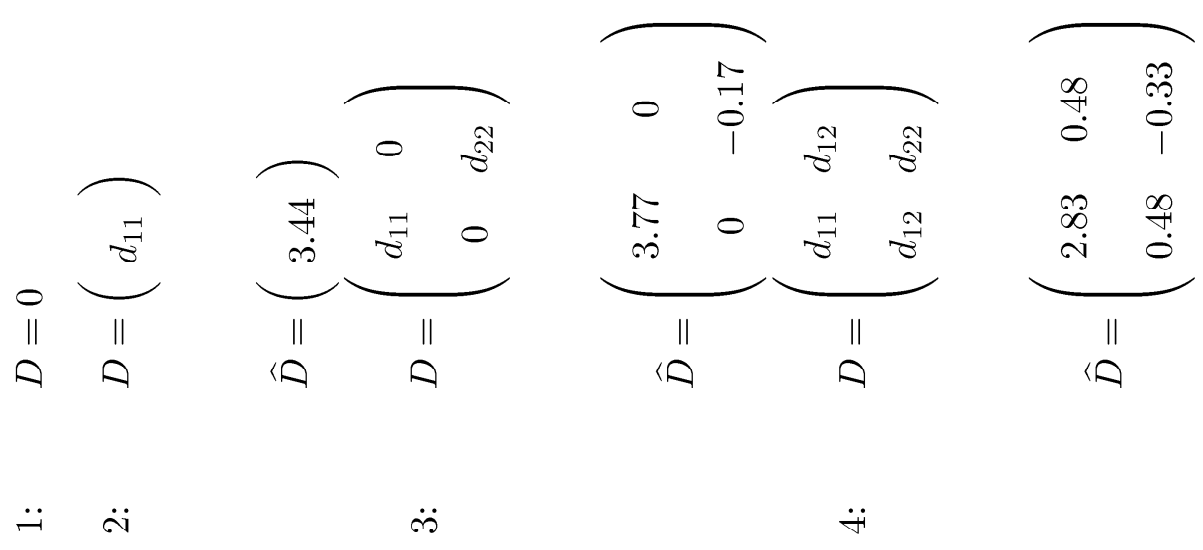



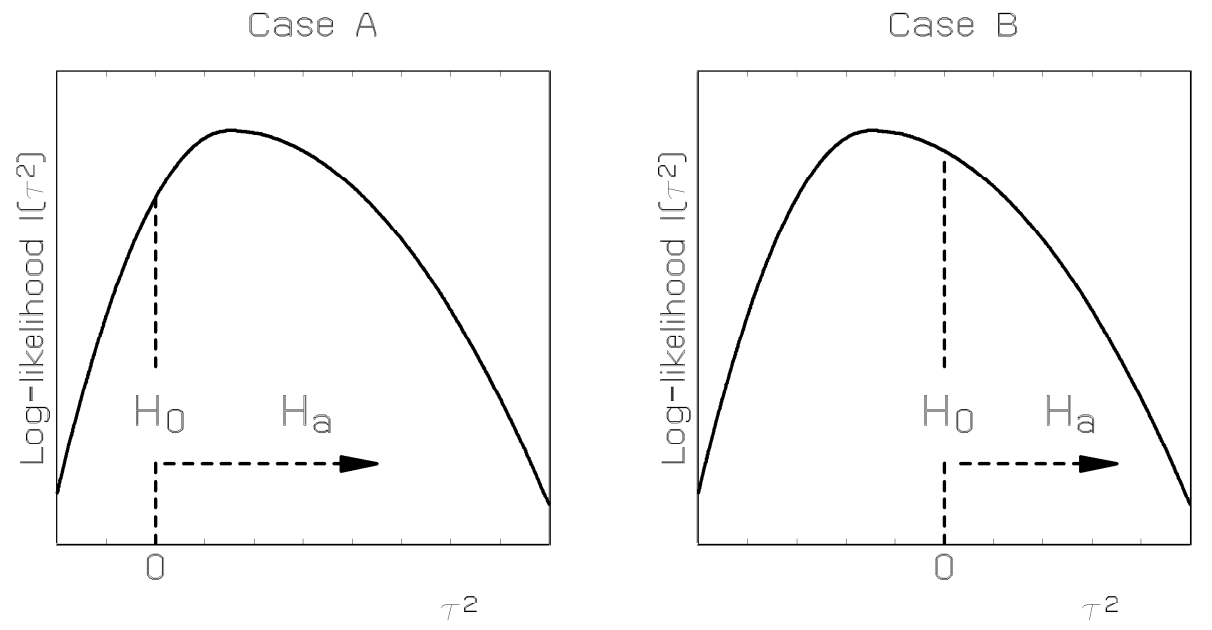
Control

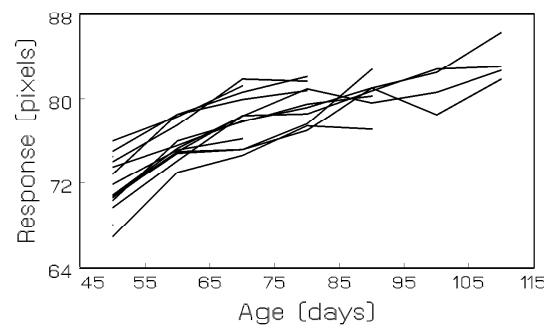

High dose

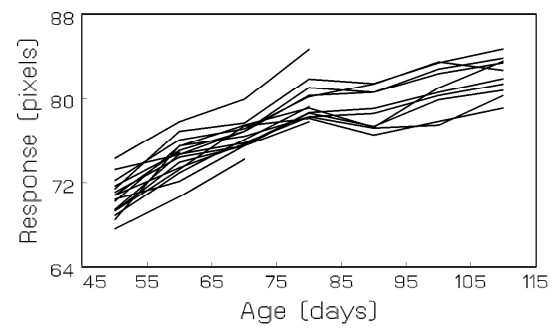

Low dose

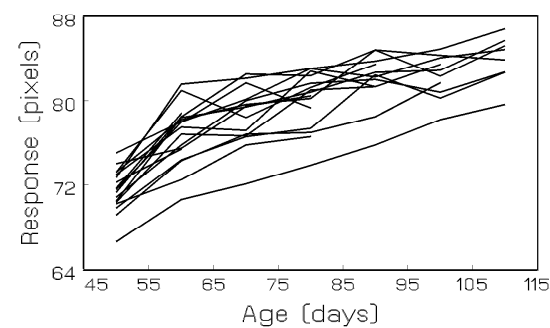

